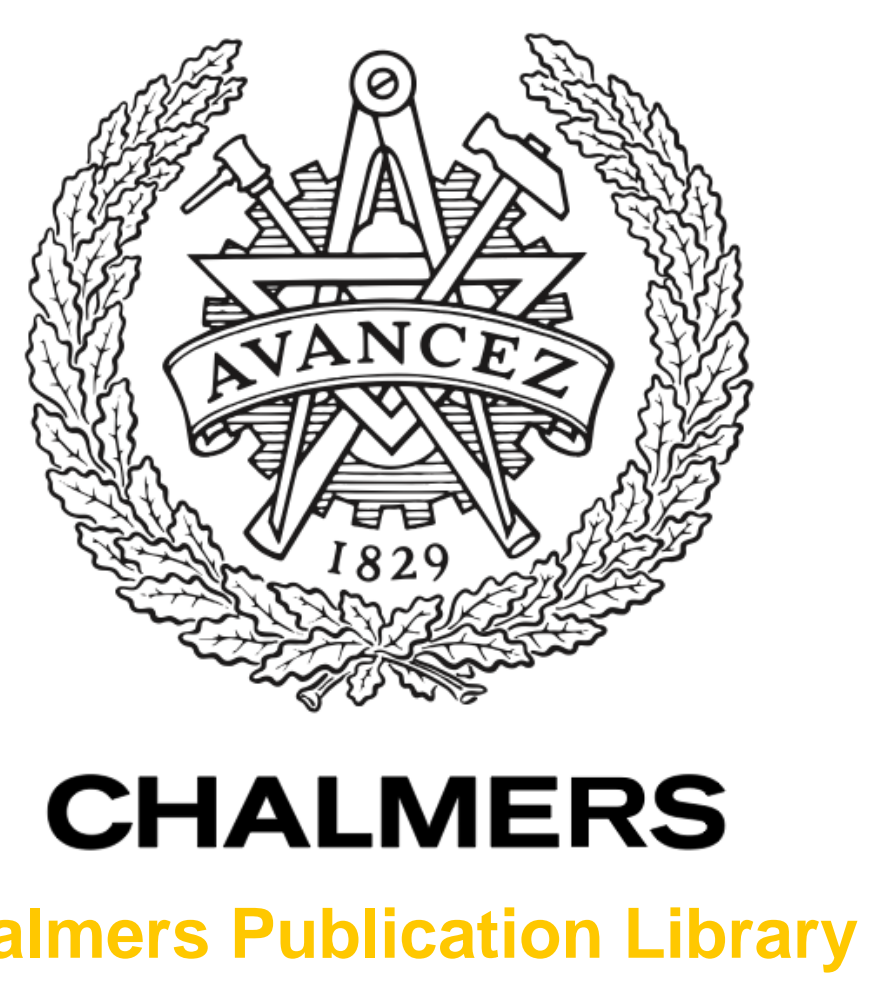

Chalmers Publication Library

Performance of Circular QAM Constellations with Time Varying Phase Noise

This document has been downloaded from Chalmers Publication Library $(\mathrm{CPL})$. It is the author's version of a work that was accepted for publication in:

IEEE 23rd International Symposium on Personal Indoor and Mobile Radio Communications (PIMRC), Sydney, 9 - 12 September 2012 (ISSN: 2166-9570)

Citation for the published paper:

Tariq, M. ; Mehrpouyan, H. ; Svensson, T. (2012) "Performance of Circular QAM

Constellations with Time Varying Phase Noise". IEEE 23rd International Symposium on

Personal Indoor and Mobile Radio Communications (PIMRC), Sydney, 9 - 12 September

2012 pp. 2365-2370.

http://dx.doi.org/10.1109/PIMRC.2012.6362752

Downloaded from: http://publications.lib.chalmers.se/publication/158320

Notice: Changes introduced as a result of publishing processes such as copy-editing and formatting may not be reflected in this document. For a definitive version of this work, please refer to the published source. Please note that access to the published version might require a subscription.

Chalmers Publication Library (CPL) offers the possibility of retrieving research publications produced at Chalmers University of Technology. It covers all types of publications: articles, dissertations, licentiate theses, masters theses, conference papers, reports etc. Since 2006 it is the official tool for Chalmers official publication statistics. To ensure that Chalmers research results are disseminated as widely as possible, an Open Access Policy has been adopted.

The CPL service is administrated and maintained by Chalmers Library. 


\section{Performance of Circular QAM Constellations with Time Varying Phase Noise}

\author{
M. Assad Tariq, \\ Department of Signals and Systems, \\ Chalmers University of Technology, \\ Gothenburg, Sweden, \\ Email: tariqm@student.chalmers.se
}

\author{
Hani Mehrpouyan, \\ Department of Signals and Systems, \\ Chalmers University of Technology, \\ Gothenburg, Sweden, \\ Email:hani.mehr@ieee.org
}

\author{
Tommy Svensson, \\ Department of Signals and Systems, \\ Chalmers University of Technology, \\ Gothenburg, Sweden, \\ Email: tommy.svensson@chalmers.se
}

\begin{abstract}
Time varying phase noise is a limiting factor in highspeed wireless communication systems, e.g., microwave backhaul links in cellular networks. This paper seeks to investigate the performance of circular M-ary quadrature amplitude modulations $(M-Q A M s)$ in the presence of time varying phase noise. A new approximate union bound expression for the symbol-error probability (SEP) of a specific circular $M-\mathrm{QAM}$ constellation is derived. Numerical results show that this expression is accurate at mediumto-high signal-to-noise ratios (SNRs) for different constellation orders, $M$. Next, exact closed-form expressions for the SEP of binary phase shift keying (BPSK) and quadrature phase shift keying (QPSK) modulations in the presence of phase noise are derived. Extensive simulations are carried out to compare the performance of rectangular and circular QAM modulations in the presence of time varying phase noise, where it is demonstrated that circular QAM modulations can outperform their rectangular counterparts when considering the effect of time varying phase noise.
\end{abstract}

\section{INTRODUCTION}

In recent years cellular communications has proven itself as an effective mean of transferring voice as well as high speed data. The demand for high speed wireless links continue to escalate with the evolution of third generation $(3 \mathrm{G})$ and fourth generation (4G) cellular networks [1]. However, the higher throughput demands by users cannot be supported without significantly increasing the data rates of the links that interconnect basestations $(B S s)$, which in turn increases the throughput requirements on the links between base station controllers (BSCs) and mobile switching centers (MSCs) or the backhaul links. BSC and MSC are interconnected using wired E1/T1 leased lines, optical fiber networks, or high-speed microwave links. Microwave radio backhauls are important for mobile operators since they can be setup much more quickly than wired-line links and due to their cost-effectiveness [2]-[4] decrease the operating and capital costs required to setup a $4 \mathrm{G}$ cellular network. As a result, in today's cellular networks more than $50 \%$ of mobile backhaul traffic is carried by point to point microwave links [5].

Traditional microwave links employ on-off keying, binary phase-shift keying (BPSK), or differential quadrature phase shift keying. On the other hand, in order to meet the stringent bandwidth efficiencies requirements for these links, the next generation microwave backhaul links are using higher order modulations, e.g., rectangular 16 or 256 quadrature amplitude

This research is in part supported by the Swedish Agency for Innovation Systems (VINNOVA), within the project P36604-1 MAGIC, Ericsson AB, and Qamcom AB. modulations (QAMs). These modulation are more complex to implement and are prone to phase noise [6], [7]. Circular QAM constellations have been widely employed in satellite broadcasting systems. The interest on circular $M$-QAM has been due to smaller amplitude fluctuation as compared to rectangular QAM, which results in fewer amplitude levels [8]. However, the potential robustness of circular QAM constellations to phase noise has been overlooked. As a matter of fact, even though high order circular QAM can be effectively used to improve bandwidth efficiency in high speed microwave links, they have not been applied widely in such systems [9], [10]. Therefore, in this paper we seek to demonstrate the potential of circular QAM constellations in improving the bandwidth efficiency and phase noise robustness of high speed wireless links.

Different circular $M$-QAM constellation designs have been purposed for code division multiple access (CDMA) and orthogonal frequency division multiplexing (OFDM) based communication systems, e.g., [11]-[13]. Moreover, in order to improve the symbol-error probability (SEP) of a communication system, in [11]-[14] circular 16-QAM constellations that have been optimized for additive white Gaussian noise (AWGN) channels are proposed. However, since the SEP optimizations in [11][14] are carried out by ignoring the effect of phase noise, the resulting constellation have an uneven phase distribution amongst its symbols and as shown later in this paper have poor performance in the presence of phase noise.

In [15] the design of circular constellations in the presence of phase offset is investigated and a closed-form SEP for a specific circular constellation is derived. However, the results in [15] are limited to constant phase offset, where it is assumed that phase noise is constant over a set of symbols. In [16], a general expression for the SEP of 2-D modulations affected by both I/Q and phase imbalances are derived. However, the results in [16] are also limited to the constant phase offset scenario and the simulations do not provide any insight on the effect of time varying phase noise on the performance of different QAM constellations. In [17], numerical schemes are used to compare the performance of circular QAM constellations for fiber optic communication systems when considering the effect of time varying phase noise. However, due to the consideration of nonlinear fiber optic channels, the proposed modulation will not perform well in wireless communication systems. The effect of phase noise on quadrature phase shift keying (QPSK) modulation has been investigated in [18]. However, in [18], no closed-form 
expression for the SEP of QPSK modulation is provided and the investigation is not expanded to higher order modulations.

In order to improve the SEP performance of a communication system in the presence of time varying phase noise and for ease of demodulation [19], in this paper, we consider the circular QAM constellation in Fig. 1, where each symbol is $90^{\circ}$ apart from its neighboring symbols ${ }^{1}$. Throughout this paper we denote this constellation by regular circular QAM. A new approximate and general union bound expression for the SEP of this circular QAM constellation is derived. Next, new closed-form expressions for the SEP of BPSK and QPSK modulations in the presence of time varying phase noise are presented and used to verify the simulation setup in this paper. Finally, the verified simulation setup is used to compare the SEP performance of regular circular QAM in Fig. 1 against that of circular constellations in [11], [12] and the rectangular QAM constellation when considering the effect of time varying phase noise. The contributions of this paper can be summarized as follows:

- A new closed-form approximate union bound expression for the SEP of the regular circular QAM modulation in Fig. 1 is derived. This expression is shown to be very accurate at medium-to-high signal-to-noise ratios (SNRs). Note that even though this result is limited to the constellation in Fig. 1, the approach outlined here provides a framework for obtaining the union-bound expressions on the SEP of other circular QAM constellations.

- New exact and closed-form expressions for the SEP of BPSK and QPSK modulations in the presence of time varying phase noise are derived and verified using numerical results.

- Extensive simulations are carried out to compare the performance of different circular and rectangular QAM constellations in the presence of time varying phase noise. These results provide specific guidelines for designing higher order QAM constellations that are more robust to phase noise. In addition, it is demonstrated that the regular circular QAM in Fig. 1 outperforms rectangular and circular QAM constellations in [11], [12].

The paper is organized as follows: Section II outlines the system model used in this paper. In section III, an approximate and closed-form union bound expression for the SEP of the circular QAM in Fig. 1 is derived and it is validated using simulations for different constellation sizes. In Section IV, the exact closed-form SEP of BPSK and QPSK modulations in the presence of phase noise are derived. In Section V, extensive simulations are carried out to compare the performance of different constellations in the presence of phase noise. Finally, Section VI summarizes the key findings of the paper.

\section{SySTEM MODEL}

A point-to-point single antenna communication system is considered. Since line-of-sight microwave links are the main focus of this paper, AWGN channels are considered. The equivalent baseband received signal model for the $k$ th symbol can be written as

${ }^{1}$ Note that compared to a rectangular QAM, the symbols in the constellation in Fig. 1 are separated by equal and larger phase angles.

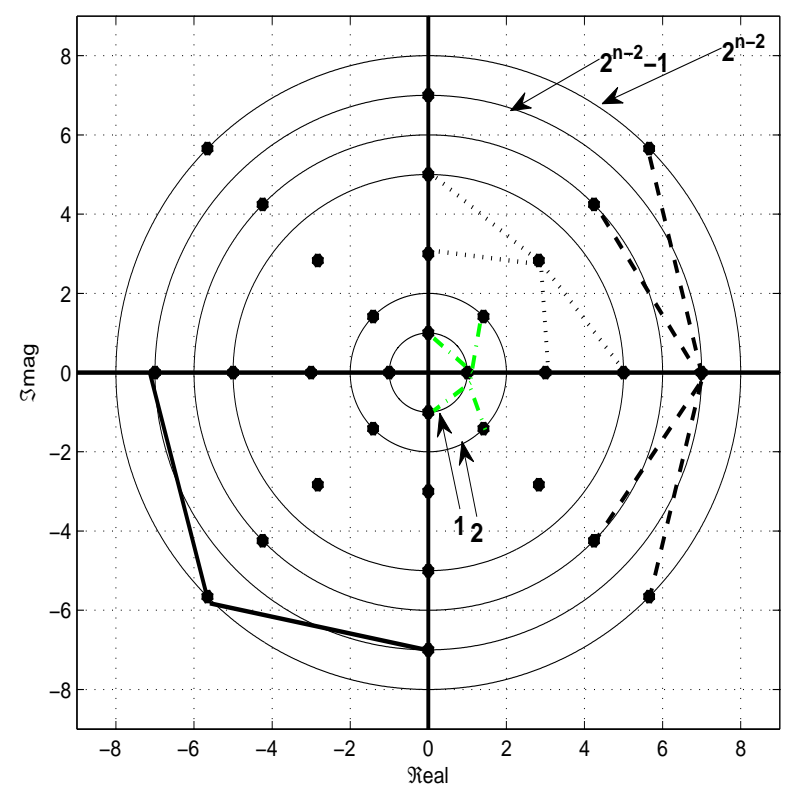

Fig. 1: Schematic illustration of the circular QAM constellation [20].

$$
r_{k}=s_{k} e^{j \theta_{k}}+w_{k}
$$

where $s_{k}$ is the $M$-ary modulated signal, where $M$ denotes the constellation size, $r_{k}$ denotes the received signal, $w_{k}$ is the zero-mean complex AWGN with variance $N_{0}$. We assume that traditional phase noise estimation algorithms, e.g., an extended Kalman filter [21] or a phase locked loop (PLL) [19], are applied at the receiver to track the phase noise of free running oscillators. Thus, $\theta_{k}$ in (1) denotes the residual phase noise corresponding to the $k$ th symbol, i.e., $\theta_{k}$ corresponds to the phase tracking error at the receiver. Thus, based on the results in [22], the phase noise process, $\theta_{k}$, is modeled as a zero-mean Gaussian random variable with variance $\sigma_{\theta_{k}}^{2}$. Note that for typical oscillators used in communication receivers the phase noise variance, $\sigma_{\theta_{k}}^{2}$, is in the range of $10^{-2}$ to $10^{-4} \operatorname{rad}^{2}$ [23].

\section{Derivation OF THE Union Bound}

Since deriving the exact closed-form SEP of the regular circular $M$-QAM in Fig. 1 is challenging, in this section an approximate closed-form union bound expression for the SEP of this constellation is derived.

In the regular circular $M$-QAM under consideration, the rings are equally spaced by a distance, $a$, and each ring has a set of four symbols that are $90^{\circ}$ apart from one another. Let us classify the rings as odd and even. Without loss of generality, it is assumed that the odd rings have their symbols positioned

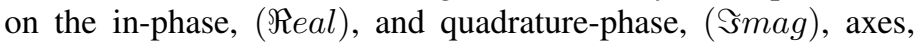
i.e., at $0^{\circ}, 90^{\circ}, 180^{\circ}$, and $270^{\circ}$, whereas the symbols on even rings are positioned at an offset of $45^{\circ}$, i.e., $45^{\circ}, 135^{\circ}, 225^{\circ}$, and $315^{\circ}$. Let $n$ denote the number of bits in a symbol, i.e., $M=2^{n}$, where it is assumed that $n \geq 3$ (note that for $n=2$ and $n=1$ the regular circular constellation in Fig. 1 simplifies to that of QPSK and BPSK, respectively, and the SEP for these constellations are available in the literature [19]). Moreover, the 
total number of rings required to accommodate $M$ symbols is calculated as $2^{n-2}$ since there are four symbols per ring. Based on Fig. 1 the even and odd rings lie at amplitudes of $A_{\text {even }}=$ $\left(2^{n-2}-2 m\right) a$ and $A_{\text {odd }}=\left(2^{n-2}-2 m-1\right) a$, respectively, where $m=0, \cdots, 2^{n-3}-1$.

At high SNR values, the AWGN variance, $N_{0}$, is small compared to symbol energy, $E_{s}$. Therefore, it can be assumed that only the neighboring symbols contribute to erroneous symbol decoding. In Fig. 1 the neighboring symbols for the outer, middle, and inner rings are highlighted. There are three specific categories of symbols that need to be taken into consideration:

- The symbols at the outer most ring (solid line in Fig. 1) have only two neighbors which lie at the $2^{n-2}-1$ th ring.

- Symbols lying on the center rings have four neighbors, these rings can be distinguished into odd rings (dashed line in Fig. 1) and even rings (dotted line in Fig. 1).

- The inner most ring (doted-dashed line in Fig. 1) have four neighbors, two on the same ring and two at immediate outer ring.

In addition, the union bound on symbol error probability, $P_{e}$, is given by [19]

where

$$
P_{e} \leq \frac{1}{M} \sum_{\substack { \mathrm{x}=1 \\
\begin{subarray}{c}{\mathrm{y}=1 \\
\mathrm{x} \neq \mathrm{y}{ \mathrm { x } = 1 \\
\begin{subarray} { c } { \mathrm { y } = 1 \\
\mathrm { x } \neq \mathrm { y } } }\end{subarray}}^{M}\left(\sqrt{\frac{|\mathrm{x}, \mathrm{y}|^{2}}{2 N_{0}}}\right),
$$

- $Q(u)=\frac{1}{\sqrt{2 \pi}} \int_{u}^{\infty} \exp \left(-\frac{v^{2}}{2}\right) d v$

- $\mathrm{x}=\left[x_{1}, x_{2} j\right]$ and $\mathrm{y}=\left[y_{1}, y_{2} j\right]$ denote the symbols, and

- $|\mathrm{x}, \mathrm{y}|$ denotes the Euclidean distance between the symbols $\mathrm{x}$ and $\mathrm{y}$, which is given by

$$
|\mathrm{x}, \mathrm{y}|=\sqrt{\left(x_{1}-y_{1}\right)^{2}+\left(x_{2}-y_{2}\right)^{2}} .
$$

Using (2) the union bound on the probability of error, $P_{e}$ can be determined as

$$
P_{e}<\frac{1}{M}\left(P_{I R}(a)+P_{C R}(a)+P_{O R}(a)\right),
$$

where $P_{\mathrm{IR}}, P_{\mathrm{CR}}$ and $P_{\mathrm{OR}}$ are the SEP for inner, center, and outer rings, respectively, and are given in $(5 \mathrm{a}),(5 \mathrm{~b})$ and $(5 \mathrm{c})$ at the bottom of this page. Note that in $(5 b) \beta(i) \triangleq 2^{n-2}-2 i$.

Remark 1: Note that by only taking into account the neighboring symbols, the SEP union bound in (4) is a looser upper bound on the SEP of the regular circular constellation in Fig. 1. However, the numerical results Fig. 2 show that the derived bound is valid for different constellation sizes at medium to high SNRs.

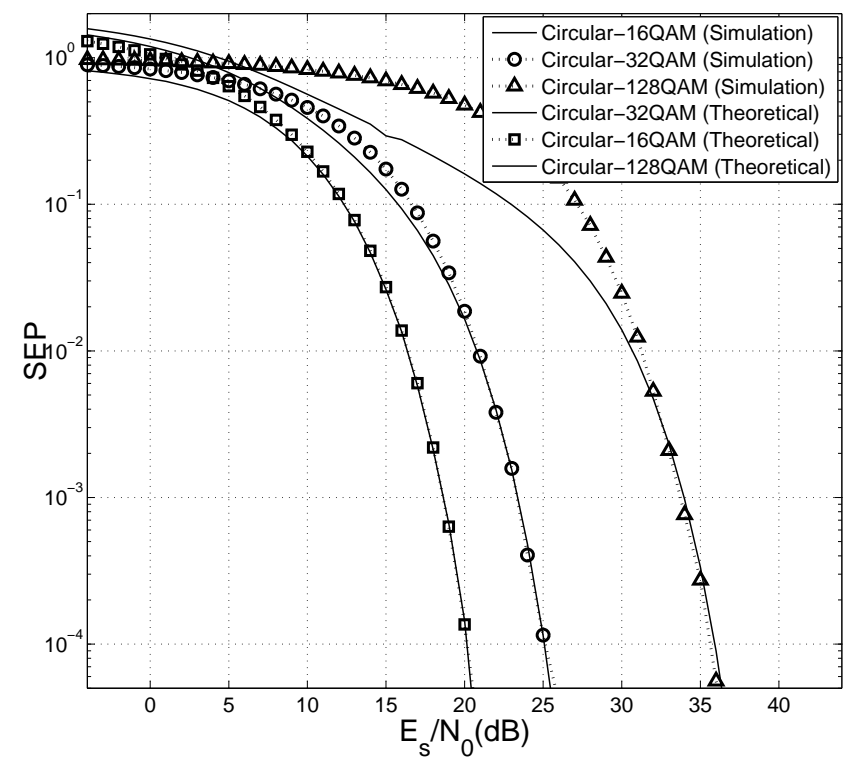

Fig. 2: SEP vs $\mathrm{E}_{\mathrm{s}} / \mathrm{N}_{0}$ in presence of AWGN for $M=$ $[16,32,128]$.

\section{EFFECT OF PHASE NOISE}

In this section the closed-form SEP expressions for BPSK and QPSK in the presence of AWGN and phase noise are presented.

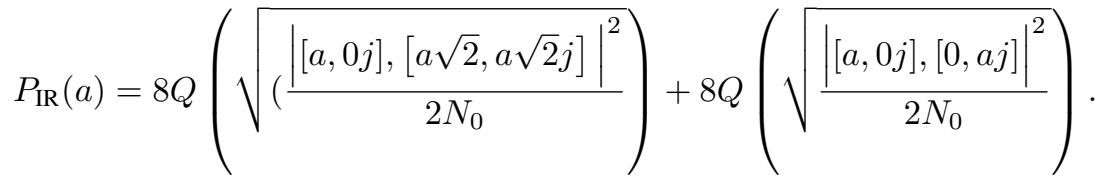

$$
\begin{aligned}
& P_{\mathrm{CR}}(a)=8 \sum_{i=1}^{2^{n-3}-1}\left\{Q\left(\sqrt{\frac{\left(\left|[(\beta(i)+1) a, 0 j],\left[(\beta(i)+2) \frac{a}{\sqrt{2}},(\beta(i)+2) \frac{a}{\sqrt{2}} j\right]\right|\right)^{2}}{2 N_{0}}}\right)\right. \\
& +Q\left(\sqrt{\frac{\left(\left|\left[\beta(i) \frac{a}{\sqrt{2}}, \beta(i) \frac{a}{\sqrt{2}} j\right],[(\beta(i)-1) a, 0 j]\right|\right)^{2}}{2 N_{0}}}\right) \\
& \left.+2 Q\left(\sqrt{\frac{\left(\left|\left[\beta(i) \frac{a}{\sqrt{2}}, \beta(i) \frac{a}{\sqrt{2}} j\right],[(\beta(i)+1) a, 0 j]\right|\right)^{2}}{2 N_{0}}}\right)\right\} . \\
& P_{\mathrm{OR}}(a)=8 Q\left(\sqrt{\frac{\left|\left[2^{n-2} \frac{\sqrt{2}}{2} a, 2^{n-2} \frac{\sqrt{2}}{2} a j\right],\left[\left(2^{n-2}-1\right) a, 0 j\right]\right|^{2}}{2 N_{0}}}\right) .
\end{aligned}
$$


Next, these closed-form expressions are applied to verify the simulation setup used for investigating the effect of phase noise on M-QAM circular and rectangular modulations.

\section{A. SEP for BPSK}

Given that the symbols $s_{1}$ or $s_{2}$ are transmitted, the conditional probability distribution function $(P D F)$ of $r_{k}$ can be written as

$$
p\left(r \mid s_{1}\right)=\frac{1}{\sqrt{\pi N_{0}}} e^{-\left|r-s_{1}\right|^{2} / N_{0}},
$$

and

$$
p\left(r \mid s_{2}\right)=\frac{1}{\sqrt{\pi N_{0}}} e^{-\left|r-s_{2}\right|^{2} / N_{0}},
$$

respectively. According to (6) and (7), the SEP given that $s_{1}$ or $s_{2}$ is transmitted can be written as

$$
P_{e}\left(r \mid s_{1}\right)=\int_{-\infty}^{0} \frac{1}{\sqrt{\pi N_{0}}} e^{-\left|r-s_{1}\right|^{2} / N_{0}} d r,
$$

and

$$
P_{e}\left(r \mid s_{2}\right)=\int_{0}^{\infty} \frac{1}{\sqrt{\pi N_{0}}} e^{-\left|r-s_{2}\right|^{2} / N_{0}} d r,
$$

respectively. Phase noise rotates the symbol along its axis. Therefore, (8) and (9) can be rewritten in terms of the phase noise parameter, $\theta_{k}$, as

$$
P_{e}\left(r \mid s_{1}\right)=\int_{-\infty}^{0} \frac{1}{\sqrt{\pi N_{0}}} e^{-\left|r-s_{1} e^{j \theta}\right|^{2} / N_{0}} d r
$$

and

$$
P_{e}\left(r \mid s_{2}\right)=\int_{0}^{\infty} \frac{1}{\sqrt{\pi N_{0}}} e^{-\left|r-s_{2} e^{j \theta}\right|^{2} / N_{0}} d r
$$

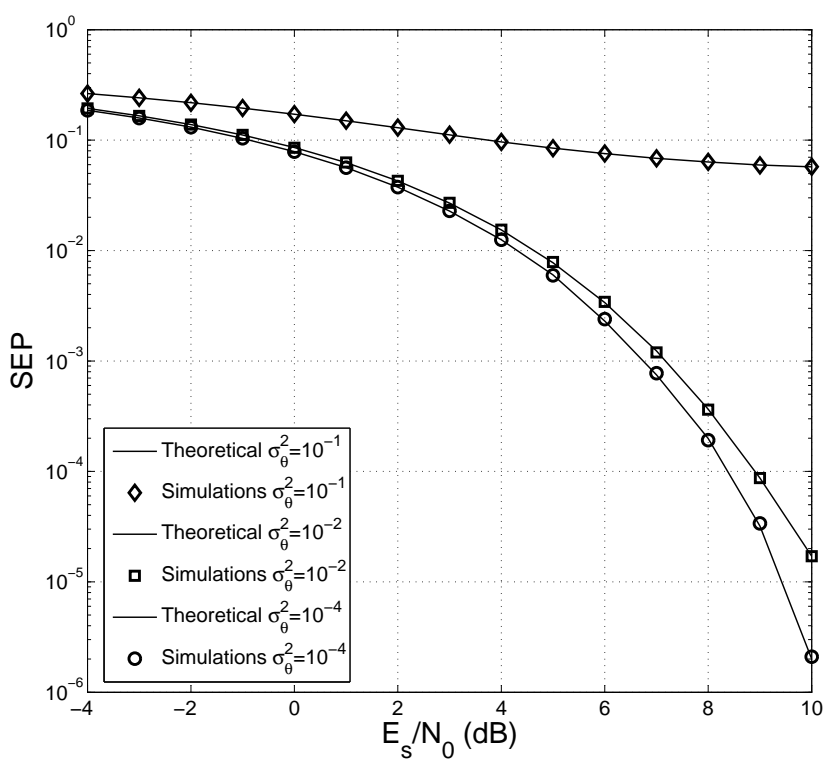

Fig. 3: SEP vs $E_{s} / N_{0}$ for BPSK for phase noise variances $\sigma_{\theta}^{2}=\left[10^{-2}, 10^{-3}, 10^{-4}\right] \mathrm{rad}^{2}$. respectively. Subsequently, the SEP for BPSK in the presence of phase noise, $P_{\mathrm{BPSK}_{\theta}}$, can be determined as

$$
P_{\mathrm{BPSK}_{\theta}}=\frac{1}{2} \operatorname{erfc}\left(\operatorname{Re}\left(e^{j \theta}\right) \sqrt{\frac{E s}{N_{0}}}\right),
$$

where

$$
\operatorname{erfc}(u)=\frac{2}{\sqrt{\pi}} \int_{u}^{\infty} e^{-u^{2}} d u .
$$

$\operatorname{Re}\left(e^{j \theta}\right)$ denotes the real component of the complex phase noise.

\section{B. SEP for QPSK}

A similar approach as above can be used for solving the SEP for the QPSK constellation and the resulting expression is given by

$$
\begin{aligned}
P_{\mathrm{QPSK}_{\theta}=} & \frac{1}{2} \operatorname{erfc}\left((\cos \theta-\sin \theta) \sqrt{\frac{E_{s}}{2 N_{0}}}\right) \\
& +\frac{1}{2} \operatorname{erfc}\left((\cos \theta+\sin \theta) \sqrt{\frac{E_{s}}{2 N_{0}}}\right) \\
+ & \frac{1}{4} \operatorname{erfc}\left((\cos \theta+\sin \theta) \sqrt{\frac{E_{s}}{2 N_{0}}}\right) \\
& \times \operatorname{erfc}\left((\cos \theta-\sin \theta) \sqrt{\frac{E_{s}}{2 N_{0}}}\right) .
\end{aligned}
$$

Remark 2: The Monte Carlo simulation results in Figs. 3 and 4 verify the closed-form SEP expressions for BPSK and QPSK modulations in (12) and (13), respectively, for different phase noise variances, $\sigma_{\theta}^{2}$. It is shown that the derived closed-form expressions for BPSK and QPSK are valid over a wide range of SNR values and overlap the theoretical results.

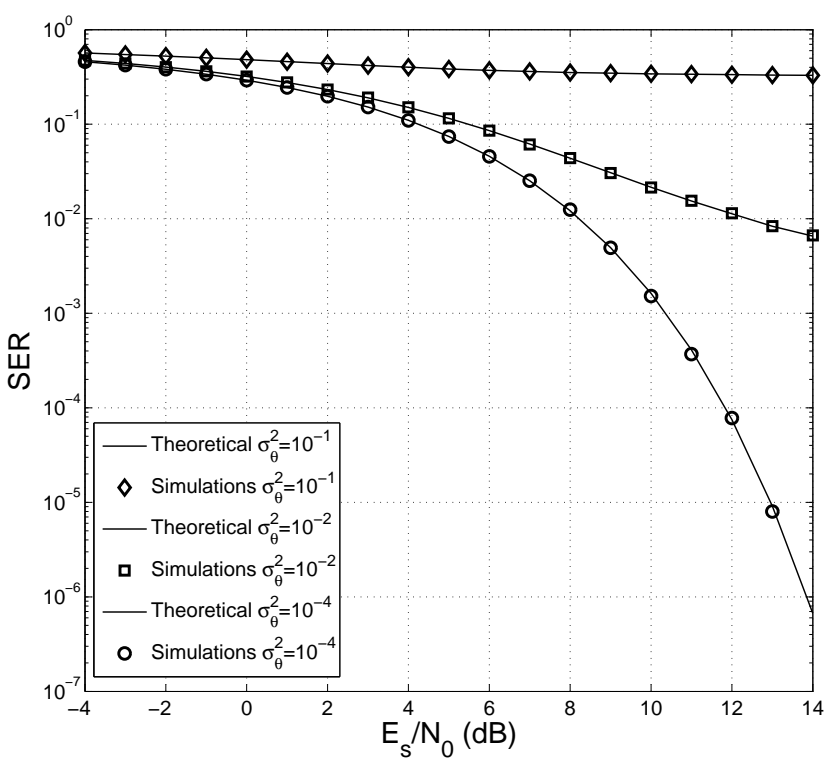

Fig. 4: SEP vs $E_{s} / N_{0}$ for QPSK for phase noise variances $\sigma_{\theta}^{2}=\left[10^{-2}, 10^{-3}, 10^{-4}\right] \mathrm{rad}^{2}$. 


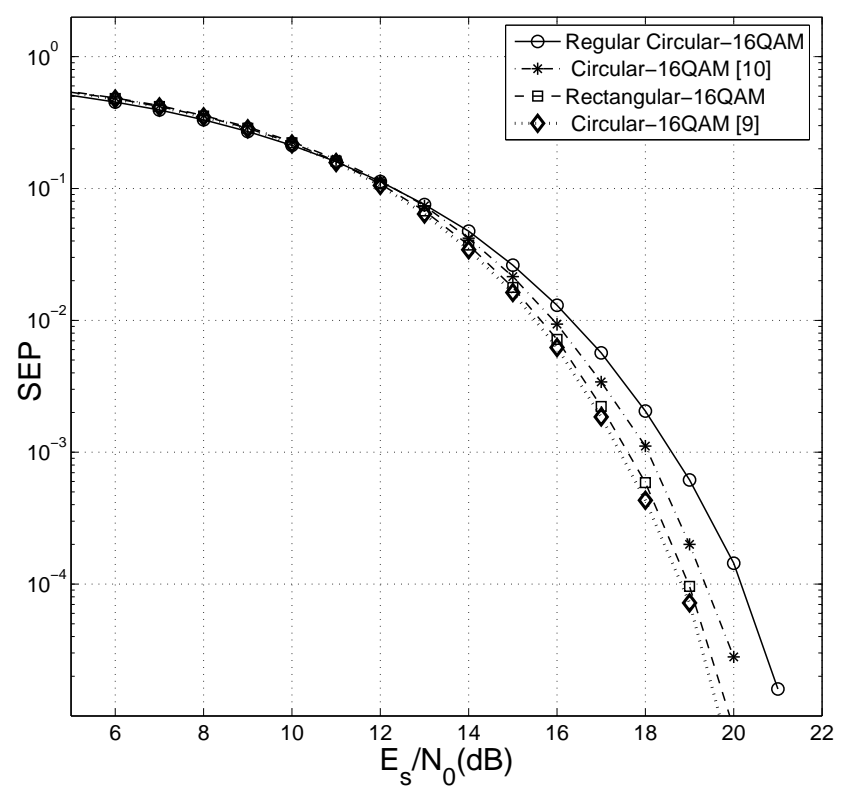

Fig. 5: Comparison of the SEP performance of regular circular 16QAM, the circular 16QAMs in [11], [12], and rectangular 16QAM when ignoring the effect of phase noise.

\section{Numerical AND Simulation Results}

Throughout this section Monte-Carlo simulations are used to investigate the SEP performance of regular circular 16-QAM, the circular 16-QAM in [11], the circular-16QAM in [12], and rectangular 16-QAM constellations in the presence of phase noise.

Fig. 5 depicts the SEP performance of the regular circular 16-QAM and that of [11], [12], and rectangular 16-QAM when ignoring the effect of phase noise. It is observed that at an SEP of $10^{-5}$, the constellations in [11], [12], and rectangular-QAM outperform the regular circular 16-QAM outlined in this paper by $2 \mathrm{~dB}$. This is anticipated, since the circular constellations in [11], [12] are optimized for AWGN channels while ignoring the effect of phase noise. Therefore, as shown in 5, they outperform the regular circular 16-QAM used in this paper. However, due to this optimization and the fewer number of rings and amplitude levels, each ring within the constellations in [11], [12] has a larger number of symbols, which in turn reduces the phase gap between symbols. In comparison, the regular circular 16-QAM depicted in Fig. 1 has wider and equal phase error free regions for each symbol (each symbol is $90^{\circ}$ apart from adjacent symbols). Furthermore, it can be noted that in the case of rectangular 16QAM the symbols on the center rectangles have a smaller phase difference compared to the most inner and outer rings. As a result, it is expected that they would significantly contribute to the SEP in the presence of phase noise.

In Figs. 6, 7, and 8 phase noise is also introduced to the communication system. Monte Carlo simulation is performed on aforementioned modulation schemes for different phase noise variances. Fig. 6,7 , and 8 show that phase noise appears to decrease the dynamic range of the SEP, since it rotates the symbols and reduces the overall error free region for each

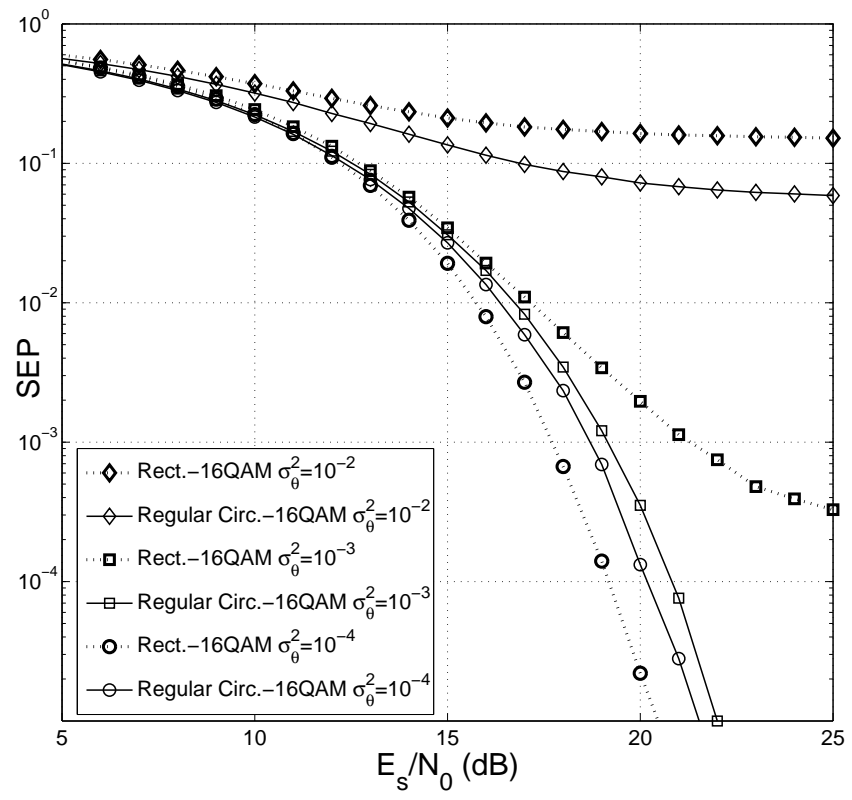

Fig. 6: SEP of regular circular 16QAM vs. rectangular 16QAM in the presence of phase noise.

symbol, in turn resulting in higher SEPs for all the modulations under consideration. It is also observed that the regular circular 16QAM constellation outlined in this paper, performs $4 \mathrm{~dB}$ better than the rectangular 16QAM at intermediate phase noise variances, i.e., $\sigma_{\theta}^{2}=\left[10^{-2}, 10^{-3}\right] \mathrm{rad}^{2}$. In addition, note that at an SEP of $10^{-4}$ and phase noise variance of $\sigma_{\theta}^{2}=10^{-3} \mathrm{rad}^{2}$, the overall SEP for a communication system employing rectangular 16QAM does not improve by increasing the SNR whereas the regular circular 16QAM in Fig. 1 continues to perform better as the SNR increases. This demonstrates the potential of the regular circular QAM constellation outlined in this paper in improving the performance of communication systems when phase noise is a dominating factor, e.g., point-to-point high-speed microwave links [9].

Figs. 7, and 8 also show that regular circular 16QAM also outperforms the circular 16QAM constellations in [11] and [12] at intermediate phase noise rates. This can be anticipated since these constellations have been designed by ignoring the effect of phase noise. This result demonstrates the importance of considering the effect of AWGN and other impairments such as phase noise when designing a modulation for high-speed communication systems. Finally, we would like to point out that the regular circular QAM constellation in this paper can be altered (by changing the number of symbols on the outer or inner rings) to outperform the rectangular QAM for any specific phase noise variance through an exhaustive search. However, this is the subject our current research and beyond the scope of this paper.

VI. CONCLUSION

In this paper, a closed-form expression for the SEP union bound of the regular circular $M$-QAM in Fig. 1 is derived. Numerical results demonstrated that the derived SEP bound is accurate at medium-to-high SNRs. Next, the exact closedform SEP expressions of BPSK and QPSK in the presence of 


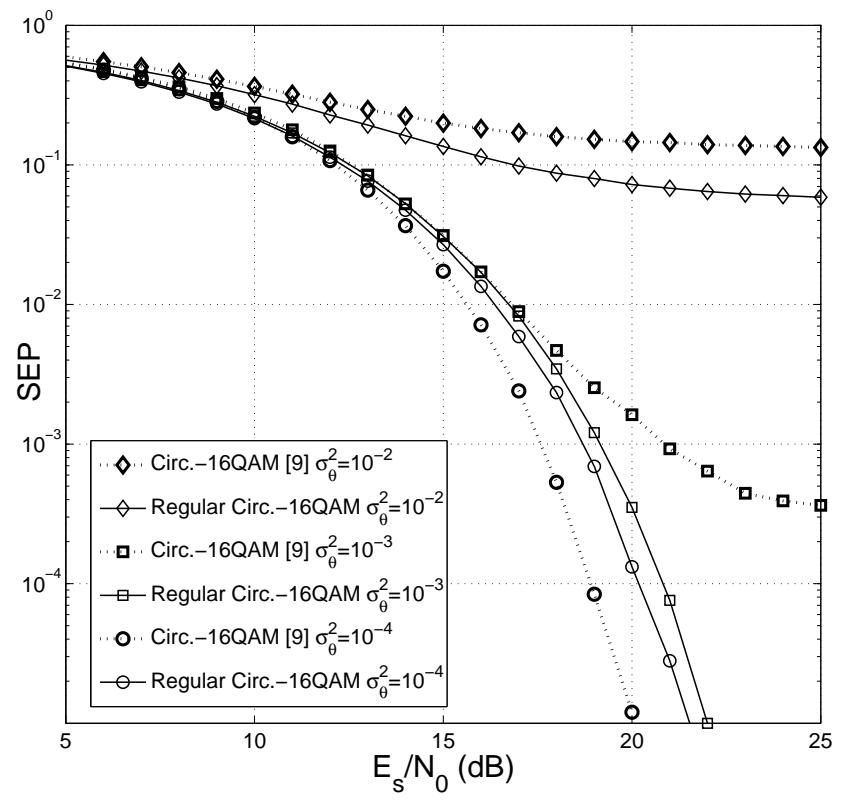

Fig. 7: SEP of regular circular 16QAM vs. circular 16QAM in [11] in the presence of phase noise.

phase noise are derived and these expressions are shown to be accurately determining the SEP of these constellations over a wide range of SNRs and phase noise variances. Extensive simulations demonstrate that the considered regular circular 16QAM outperforms existing circular and rectangular 16-QAM constellations at intermediate phase noise variances by an average of $4 \mathrm{~dB}$. Note that this constellation can be altered to outperform the rectangular $M$-QAM constellations for any specific phase noise variance and SNR through an exhaustive search. However, finding a systematic and low complexity method for determining such circular constellations is subject of current research.

\section{ACKNOWLEDGEMENT}

The authors would like to thank Tryggve Mathiesen and Dag Mortensen at BitSim AB whose guidance has been helpful in shaping the overall scope of this article.

\section{REFERENCES}

[1] E. Boch, "High-capacity ethernet backhaul radio systems for advanced mobile data networks," IEEE Microwave Mag., vol. 10, no. 5, pp. 108114, Aug. 2009.

[2] F. Ivanek, "Mobile backhaul," IEEE Microwave Mag., vol. 10, no. 5, pp. 10-20, Aug. 2009.

[3] S. Chia et. al, "3G evolution," IEEE Microwave Mag., vol. 9, no. 4, pp. 52-63, Aug. 2008.

[4] D. Rouffet and P. Sehier, "Convergence and Competition on the Way Towards 4G," in IEEE Radio and Wireless Symp., Jan. 2007.

[5] S. Little, "Is microwave backhaul up to the 4G task?" IEEE Microwave Mag., vol. 10, no. 5, pp. 67-74, Aug. 2009.

[6] Z. He, J. Chen, Y. Li, and H. Zirath, "A Novel FPGA-Based 2.5Gbps DQPSK Modem for High Capacity Microwave Radios," in Proc. IEEE Int. Conf. on Commun., May 2010.

[7] M. R. Khanzadi, H. Mehrpouyan, E. Alpman, D. Kuylenstierna, T. Eriksson, and T. Svensson, "On models, bounds, and estimation algorithms for timevarying phase noise," in Proc. Int. Conf. Signal Process. and Commun. Systems (ICSPCS), Dec. 2011.

[8] Y. Li, S. Xu, and H. Yang, "Design of Circular Signal Constellations in the Presence of Phase Noise," in Proc. Int. Conf. on Wireless Commun., Networking and Mobile Computing, Oct. 2008.

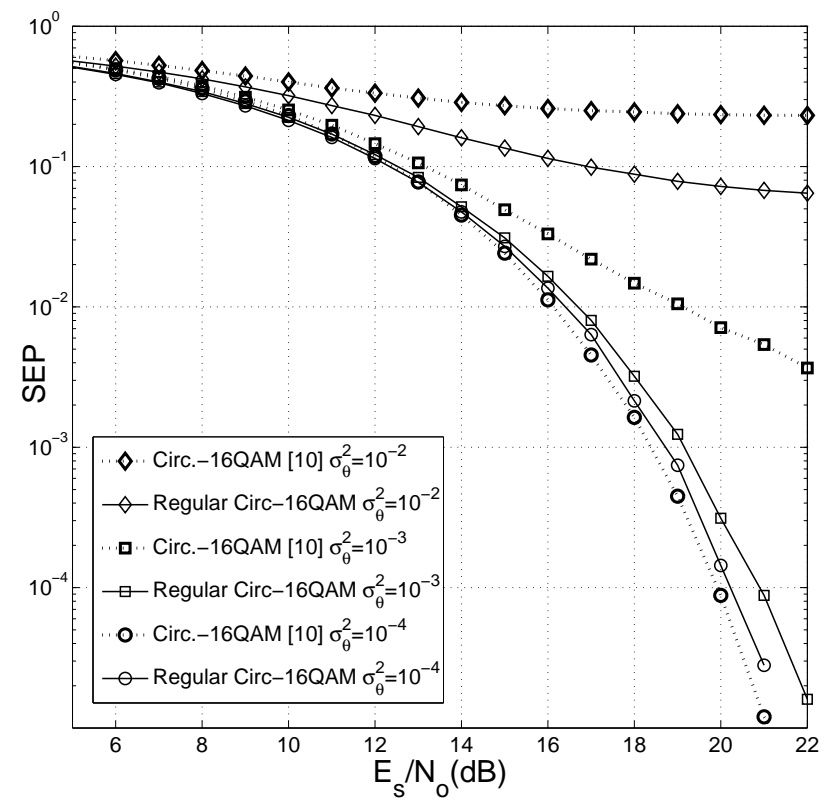

Fig. 8: SEP of regular circular 16QAM vs. circular 16QAM in [12] in the presence of phase noise.

[9] J. Wells, Multi-Gigabit Microwave and Millimeter-Wave Wireless Communications. Artech House, 2010.

[10] H. Mehrpouyan, A. A. Nasir, T. Eriksson, S. D. Blostein, G. K. Karagiannidis, and T. Svensson, "Joint estimation of channel and oscillator phase noise in MIMO systems," accepted for pulication in IEEE Trans. Signal Process., May 2012.

[11] K. Abdullah, A. F. Ismail, W. Hashim, and Z. M. Hussain, "An optimal circular 16-QAM modulation technique for wavelet and Fourier based OFDM," in Proc. Int. Conf. on Computer and Commun. Engineering, May 2010.

[12] S. Cheung, "A $(4,12)$ circular signal set for satellite transmission," in Proc. IEE National Conf. on Telecommunications, Apr. 1989.

[13] J. Dong, Y. Zou, and D. Li, "Optimal 2-Circular 16QAM constellation design," in Proc. IEEE Personal, Indoor and Mobile Radio Commun., vol. 3, Sep. 2003.

[14] K. Abdullah, S. M. Saeedahmed, and Z. M. Hussain, "Performance analysis of an optimal circular 16-QAM for wavelet based ofdm systems," in Int. J. Communications, Network and System Sciences, Dec 2009.

[15] Y. Li, S. Xu, and H. Yang, "Design of circular signal constellations in the presence of phase noise," in Proc. Int. Conference on Wireless Commun., Networking and Mobile Computing, Oct. 2008.

[16] J. Lee, D. Yoon, and I. C. Im, "General expression for the error probability of arbitrary two-dimensional signaling with I/Q amplitude and phase unbalances," in Proc. Int. Conf. Advanced Communication Technology, pp. 1781-1784, Feb. 2006

[17] S. Tharranetharan, M. Saranraj, S. Sathyaram, and V. R. Herath, "A performance comparison of nonlinear phase noise tolerant constellation diagrams," in Proc. IEEE Int. Conf. on Industrial and Infor. Systems, pp. 439-442, Aug. 2011.

[18] D. Taggart and R. Kumar, "Impact of phase noise on the performance of the QPSK modulated signal," in Proc. IEEE Aerospace Conference, Mar. 2011.

[19] J. G. Proakis and M. Salehi, Digital Communications, 5th ed. McGraw-Hill International, 2008

[20] S. Bergmann and D. Taylor, "Error-rate for the 9600 bps signalling configuration of the V.29 modem," Canadian Elect. Eng. J., vol. 6, no. 1, pp. 16-19, Jan. 1981

[21] S. A. Merritt, "The iterated extended Kalman phase detector," IEEE Trans. Commun., vol. 37, no. 5, pp. 522-526, May 1989.

[22] S. M. Kay, Fundamentals of Statistical Signal Processing, Estimation Theory. Prentice Hall, Signal Processing Series, 1993.

[23] A. Hajimiri, S. Limotyrakis, and T. H. Lee, "Jitter and phase noise in ring oscillators," IEEE J. Solid-State Circuits, vol. 34, no. 6, pp. 790-804, Jun. 1999. 